Fowler MD, Neuropediatric Unit, Karolinska University Hospital, 17176 Stockholm, Sweden. E-mail: asa.fowler@ki.se).

COMMENT. Recovery from acute encephalitis in children is incomplete in $66 \%$ and complete in $34 \%$ cases at long-term follow-up. Many of those considered fully recovered at discharge have persisting symptoms later. Those who recover completely do so within 1 year. Ten percent develop post-encephalitic epilepsy, and girls are especially vulnerable. In addition to EEG abnormalities, neurological and MRI findings at time of acute illness are predictive of outcome. (Lee WT et al. Eur J Ped Neur 2007;1 1(5):302309)(Chen Y-J et al. J Child Neurol 2006;21:1047-1051).

\title{
ANTI-NMDA RECEPTOR ENCEPHALITIS AND PROLONGED NONCONVULSIVE STATUS EPILEPTICUS
}

A case of a 35-year-old woman with a 3 week history of headaches, short-term memory loss, and psychosis, diagnosed with anti-NMDA receptor encephalitis and ovarian tumor, is reported from the University of Rochester Medical Center, NY, and University of Pennsylvania Medical Center, Philadelphia. An EEG demonstrated nonconvulsive status epilepticus (NCSE). MRI showed hyperintensity in the right medial temporal lobe. Tests for viral and bacterial pathogens, including herpes simplex virus, were negative. CSF had an antibody for the NR1/NR2B heteromer of the NMDA receptor. Most AEDs were ineffective but propofol caused abrupt cessation of the rhythmic NCSE. Pentobarbital coma was required to maintain EEG-burst suppression and was continued for 5 months. IV immunoglobulin, cyclophosphamide, or rituximab were without effect. CTs and ultrasound of ovaries revealed only a cyst, but oopherectomy at 5 months uncovered an ovarian teratoma. Two weeks postoperatively she awakened, and within 4 weeks she was alert and conversant. At 5 weeks postoperativly, the EEG showed sleep-wake cycles and normal waking organization. Mild defects on naming and memory tests were present at 6 months follow-up, but no seizures had occurred. (Johnson N, Henry C, Fessler AJ, Dalmau J. Anti-NMDA receptor encephalitis causing prolonged nonconvulsive status epilepticus. Neurology Oct 2010;75:1480-1482). (Response and reprints: Dr Nicholas Johnson, 601 Elmwood Ave, Box 673, Rochester, NY 14642. E-mail: Nicholas.johnson@urmc.rochester.edu).

COMMENT. Anti-NMDA receptor encephalitis resistant to immonomodulatory therapies should be considered for oopherectomy, even when CT is not diagnostic of ovarian tumor.

\section{DEMYELINATING DISEASES}

\section{PROGRESSIVE COGNITIVE IMPAIRMENTS IN CHILDHOOD AND JUVENILE MULTIPLE SCLEROSIS}

The evolution of cognitive and psychosocial difficulties in a cohort of 56 MS patients compared with 50 healthy controls was studied by researchers at the University 
of Florence and centers in Milan, Bari, Catania, and Rome, Italy. At a mean period of 2 years follow-up, $70 \%$ had cognitive impairment, deteriorating in $75 \%$, especially verbal memory, attention, verbal fluency, and receptive language. Older age was a predictor of cognitive deterioration $(\mathrm{p}=0.003)$. Depression developed in $30 \%$, fatigue in $21 \%$, and interference with school activities in $30 \%$. Regular assessment of psychosocial functioning and rehabilitation strategies in MS childhood patients are indicated. (Amato MP, Goretti B, Ghezzi A et al. Cognitive and psychosocial features in childhood and juvenile MS: Two-year follow-up. Neurology Sept 28, 2010;75:1134-1140). (Response and reprints: Dr Maria Pia Amato, Department of Neurology, University of Florence, Viale Morgagni 85, 50134 Florence, Italy. E-mail: mariapia.amato@unifl.it).

COMMENT. In a previous multicenter study, these investigators found a $31 \%$ prevalence of cognitive impairment and a low IQ in $28 \%$ of 63 childhood MS cases. (Amato MP et al. Neurology 2008;70:1891-1897).

\section{COGNITIVE LEISURE AND COGNITIVE RESERVE IN MULTIPLE SCLEROSIS PATIENTS}

The effect of cognitive leisure (eg reading books, playing cards etc) on prevention of cognitive impairment was studied in 36 adults (31women) with MS treated at Kessler Research Center, West Orange, New Jersey Medical School, and Teachers College, Columbia University, NY, NY. Cognitive status was measured with a composite score of processing speed and memory, and brain atrophy was measured by third ventricle width on MRI. Controlling for brain atrophy, vocabulary and education, premorbid cognitive leisure was positively associated with current cognitive status $(\mathrm{p}<0.01)$. Patients with MS who engaged in more cognitive leisure were able to withstand the effects of more severe brain atrophy. Cognitive leisure is an independent source of cognitive reserve in patients with MS. (Sumowski JF, Wylie GR, Gonnella A, Chiaravalloti N, DeLuca J. Premorbid cognitive leisure independently contributes to cognitive reserve in multiple sclerosis. Neurology Oct 19,2010;75:1428-1431). (Response and reprints: Dr James F Sumowski, Neuropsychology \& Neuroscience Laboratory, Kessler Foundation Research Center, 300 Executive Drive, Suite 10, West Orange, NJ 07052. E-mail: isumowski@kesslerfoundation_org).

COMMENT. Lifetime intellectual enrichment lessens the adverse effect of neurologic disease (eg Alzheimer disease) on cognitive status. (Stern Y. Neuropsychologia 2009;47:2015-2028). The present study shows that cognitive leisure time may contribute to cognitive reserve in patients with MS.

The "London taxi driver" study provides neuroanatomic support for the cognitive reserve hypothesis (Maguire EA et al. Navigation-related structural change in the hippocampi of taxi drivers. Proc Natl Acad Sci USA 2000;97:4398-4403). The time as a taxi driver (in London) is positively correlated with posterior hippocampal volumes (serving spatial memory). I believe that London taxi drivers were required to study and pass a City address and navigation test to obtain a license. Not an easy task. The term "cognitive toil" might be more appropriate. 